\title{
Speed of sound reconstruction for HIFU ultrasound thermometry using an ultrasound element: simulation study (Withdrawal Notice)
}

Younsu Kim, Chloé Audigier, Nicholas Ellens, Emad Boctor

Younsu Kim, Chloé Audigier, Nicholas Ellens, Emad M. Boctor, "Speed of sound reconstruction for HIFU ultrasound thermometry using an ultrasound element: simulation study (Withdrawal Notice)," Proc. SPIE 10580, Medical Imaging 2018: Ultrasonic Imaging and Tomography, 1058013 (13 April 2018); doi: $10.1117 / 12.2293141$ 


\section{Speed of sound reconstruction for HIFU ultrasound thermometry using an ultrasound element: simulation study}

Younsu Kim, Chloe Audigier, Nicholas Ellens and Emad M. Boctor

Johns Hopkins University (United States)

Proc. SPIE 10580, 1058013 (2018)

Online Publication Date: 13 April 2018

Withdrawn from Publication: 25 April 2018

Conference Date: 13-16 February 2018

Conference Location: San Diego, California, United States

Conference Title: Ultrasonic Imaging and Tomography

Conference Chairs: Neb Duric and Brett Byram

Publisher's Note: This paper, originally published on 13 April 2018, was withdrawn per author request. 